\title{
PERFECT CODES IN THE GRAPHS $O_{k}$ AND $L\left(O_{k}\right)$
}

\author{
by D. H. SMITH
}

(Received 26 April, 1979)

In [6] the question of the existence of perfect $e$-codes in the infinite family of distance-transitive graphs $O_{k}$ was considered. It was pointed out that it is difficult to rule out completely any particular value of $e$ because of the difficulty of working with the sphere packing condition. For $e=1,2,3$ it can be seen from the results of [6] that the condition given by the generalisation of Lloyd's theorem is satisfied for infinitely many values of $k$. We shall show that this is not the case for $e=4$ and we shall prove that there are no perfect 4 -codes in $O_{k}$.

Hammond [5] has constructed perfect 1-codes in the line graphs $L\left(O_{k}\right)$. In fact $L\left(O_{k}\right)$ contains $2 k-1$ perfect 1 -codes which form a partition of the vertex set of $L\left(O_{k}\right)$. We show that the codes described by Hammond are unique.

Definition. The graph $O_{k}(k \geqslant 2)$ has $\left(\begin{array}{c}2 k-1 \\ k-1\end{array}\right)$ vertices indexed by the $(k-1)$-subsets of the set $\{1,2, \ldots, 2 k-1\}$. Two vertices are joined by an edge if and only if their indexing sets are disjoint.

Definitron. The line graph $L\left(O_{k}\right)$ has vertices which correspond to the edges of $O_{k}$, with two vertices of $L\left(O_{k}\right)$ being adjacent whenever the corresponding edges of $O_{k}$ are incident.

Definition. A perfect $e$-code in a graph $\Gamma$ is a subset $C$ of the vertices of $\Gamma$ with minimum distance $2 e+1$ such that any vertex of $\Gamma$ is at distance at most $e$ from some vertex of $C$. We consider only nontrivial codes $(|C|>2)$.

Definition. Define the sequence of polynomials $\left\{v_{i}(\lambda)\right\}$ by $v_{0}(\lambda)=1, v_{1}(\lambda)=\lambda$, $c_{i+1} v_{i+1}(\lambda)-\lambda v_{i}(\lambda)+b_{i-1} v_{i-1}(\lambda)=0 \quad$ where $\quad c_{i}=\left[\frac{1}{2}(i+1)\right] \quad$ and $\quad b_{i}=k-\left[\frac{1}{2}(i+1)\right]$ $(i=1,2, \ldots, d-1)$. Let

$$
x_{j}(\lambda)=\sum_{i=0}^{j} v_{i}(\lambda) .
$$

The following lemma is the generalisation of Lloyd's theorem.

LEMMA 1. [4], [6]. If $O_{k}$ contains a perfect $e$-code, then the roots of $x_{e}(\lambda)$ are members of the set $\left\{-(k-1),(k-2),-(k-3), \ldots,(-1)^{k+1}\right\}$.

Lemma 2 [6]. If $O_{k}$ contains a nontrivial perfect $e$-code, then $k \geqslant\left(e^{2}+4 e+2\right) / 2(e$ even) and $k \geqslant\left(e^{2}+4 e+3\right) / 2(e$ odd $)$.

Lemma 3 [6]. If $\alpha \neq-1$ is a root of $x_{e}(\lambda)$, then so is $-\alpha-1$. If $e$ is $o d d,-1$ is a root of $x_{e}(\lambda)$. 
Now let $\bar{x}_{e}(\lambda)=c x_{e}(\lambda)$ (c constant) be a monic polynomial and consider $\bar{x}_{e}\left(-\frac{1}{2}\right)$ which is a polynomial in $k$ of degree [e/2]. (The use of the polynomial $\bar{x}_{e}\left(-\frac{1}{2}\right)$ was suggested by E. Bannai.)

Consider the case $e=4$. Suppose the roots of $x_{4}(\lambda)$ are $\alpha_{1}, \alpha_{2},-\alpha_{1}-1,-\alpha_{2}-1$.

$$
\bar{x}_{4}\left(-\frac{1}{2}\right)=\left(-\frac{1}{2}-\alpha_{1}\right)\left(-\frac{1}{2}-\alpha_{2}\right)\left(-\frac{1}{2}+\alpha_{1}+1\right)\left(-\frac{1}{2}+\alpha_{2}+1\right)
$$

and so we have

$$
16 \bar{x}_{4}\left(-\frac{1}{2}\right)=\left(2 \alpha_{1}+1\right)^{2}\left(2 \alpha_{2}+1\right)^{2}=w^{2} .
$$

Straightforward calculation reveals that

$$
x_{4}(\lambda)=\left[\lambda^{4}+2 \lambda^{3}+\lambda^{2}(7-4 k)+\lambda(6-4 k)+2(k-1)(k-2)\right] / 4
$$

and so the equation (1) becomes

$$
32 k^{2}-80 k+41=y^{2}
$$

Also since

$$
\begin{aligned}
7-4 k= & \alpha_{1}\left(-\alpha_{1}-1\right)+\alpha_{1} \alpha_{2}+\alpha_{1}\left(-\alpha_{2}-1\right)+\left(-\alpha_{1}-1\right) \alpha_{2} \\
& +\left(-\alpha_{1}-1\right)\left(-\alpha_{2}-1\right)+\alpha_{2}\left(-\alpha_{2}-1\right) \\
= & -\alpha_{1}\left(\alpha_{1}+1\right)-\alpha_{2}\left(\alpha_{2}+1\right)+1,
\end{aligned}
$$

we have

$$
4 k-6=\alpha_{1}\left(\alpha_{1}+1\right)+\alpha_{2}\left(\alpha_{2}+1\right)
$$

and

$$
2(k-1)(k-2)=\alpha_{1}\left(\alpha_{1}+1\right) \alpha_{2}\left(\alpha_{2}+1\right) .
$$

Hence $\left.\alpha_{1}\left(\alpha_{1}+1\right)=2 k-3 \pm \sqrt{\left(2 k^{2}-6 k+5\right.}\right)$. Since $\alpha_{1}$ is an integer, $2 k^{2}-6 k+5$ is a perfect square and so

$$
32 k^{2}-96 k+80=z^{2} .
$$

Hence if a perfect 4-code exists in $O_{k}$, equations (2) and (3) have a simultaneous integer solution and from Lemma $2, k \geqslant 17$. From (2) and (3) we have $16 k-39=y^{2}-z^{2}$. Write $z=\gamma k>0$. Then $(\gamma k)^{2}=32 k^{2}-96 k+80$ gives $\gamma<4 \sqrt{2}<6$ and $k \geqslant 17$ gives $(\gamma k)^{2}>25 k^{2}$ so $\gamma>5$. If we write $y=\gamma k+i$ (where $i$ is a positive integer) we have $16 k-39=2 \gamma k i+i^{2}$, so

$$
10 k i+i^{2}<16 k-39<12 k i+i^{2} .
$$

The first inequality gives $i<2$ and the second excludes $i=1$. Hence we have:

TheORem 1. There is no nontrivial perfect 4-code in $O_{k}$.

NOTE. It seems possible that a similar method would work for $e=5$. The equations replacing (2) and (3) can be written

$$
6(8 k-11)^{2}-114=p^{2}, \quad 3(k-2)^{2}+1=q^{2} .
$$


It is possible that the method of Baker and Davenport [1] will extend to this case, but the calculation is formidable.

Now consider the case of perfect 1 -codes in $L\left(O_{k}\right)$. Let $X=\{1,2, \ldots, 2 k-1\}$ and let $e-f$ be the edge of $O_{k}$ joining vertices $e$ and $f$. For any $x \in X$ let $\bar{C}_{x}=$ $\{c-d \mid\{c \cup d\}=X \backslash x\}$ and $C_{x}$ be the corresponding set of vertices in $L\left(O_{k}\right)$. Hammond [5] has shown that for each $x \in X$ the code $C_{x}$ is a perfect 1-code.

TheOREM 2. The codes $C_{x}(x \in X)$ are the only perfect 1-codes in $L\left(O_{k}\right)$.

Proof. The case $k=3$ is easily dealt with directly. Suppose $k>3$. Let $D$ be a code in $L\left(O_{k}\right)$ not isomorphic to any $C_{x}$ and let $\bar{D}$ be the corresponding set of edges in $O_{k}$. $D$ contains vertices of $C_{x}$ and $C_{y}$ for some $x, y \in X, x \neq y$. Choose $x, y, p \in C_{x}, q \in C_{y}$ with $p, q \in D$ in such a way that $p$ and $q$ are as close as possible with $x \neq y$. Let $C_{x}^{\prime}$ consist of those vertices in $L\left(O_{k}\right)$ adjacent to vertices of $C_{x}$. Let $p, a_{1}, a_{2}, \ldots, a_{n}, q$ be a path of minimum length joining $p$ and $q$. Clearly $a_{1} \in C_{x}^{\prime}, a_{n} \in C_{y}^{\prime}$ but all possibilities for $a_{2}$ contradict the choice of $p$ and $q$ unless $n=2$.

Since $O_{k}$ has girth $6(k>3)$ we have two cases:

Case 1

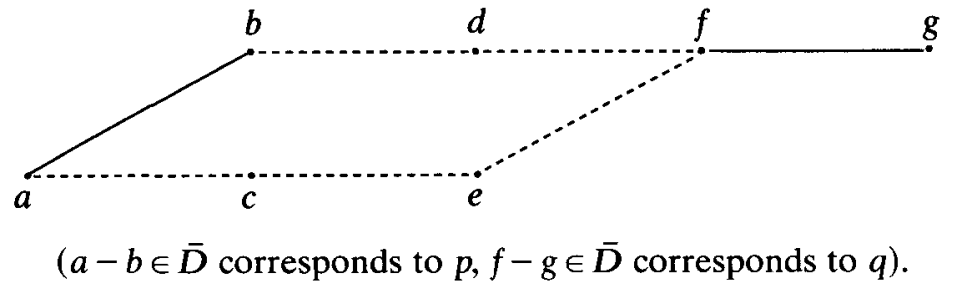

Either $c-e$ is in $\bar{D}$ or $c-e$ is adjacent to an edge of $\bar{D}$. In either case the minimum distance of $D$ would be 2 . This is a contradiction.

Case 2

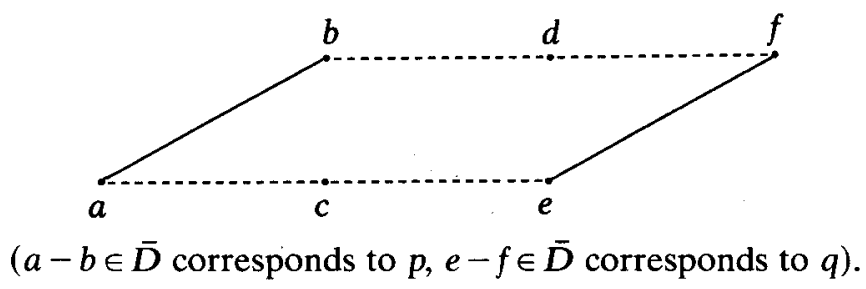

Then, rearranging $X$ if necessary, we can write without loss of generality

$$
\begin{array}{ll}
a=(12 \ldots k-1) & b=(k k+1 \ldots 2 k-2) \\
d=(23 \ldots k-1 ; 2 k-1) & c=(k+1 k+2 \ldots 2 k-1) .
\end{array}
$$

Then it is easy to see that $f=(1 ; k+1 k+2 \ldots 2 k-2), e=(23 \ldots k)$. Then $e \cup f=$ $\{X \backslash(2 k-1)\}=a \cup b$ contradicting the fact that $a-b$ corresponds to $p$ and $e-f$ corresponds to $q$. 
NoTE. Theorem 1 together with Theorem 2 of [6] show that there are no nontrivial perfect 4-codes in the graphs $2 . O_{k}$ [6]. The modifications required to the proof of Theorem 2 for the case of perfect 1-codes in the graphs $L\left(2, O_{k}\right)$ are straightforward.

\section{REFERENCES}

1. A. Baker and H. Davenport, The equations $3 x^{2}-2=y^{2}$ and $8 x^{2}-7=z^{2}$, Quart. J. Math. Oxford (2), 20 (1969), 129-137.

2. N. L. Biggs, Algebraic Graph Theory Cambridge Math. Tracts No. 67, (Cambridge University Press, London, 1974).

3. N. L. Biggs, Perfect codes and distance-transitive graphs, in "Combinatorics" (Proceedings of the British Combinatorial Conference, 1973) (Cambridge University Press, London/New York 1974), 1-8.

4. N. L. Biggs, Perfect codes in graphs, J. Combinatorial Theory, Ser. B 15 (1973), 289-296.

5. P. Hammond, $q$-coverings, codes and line graphs (to appear).

6. P. Hammond and D. H. Smith, Perfect codes in the graphs $O_{k}$.J. Combinatorial Theory, Ser. B 19 (1975), 239-255.

Polytechnic of Wales, PONTYPRIDD,

WALES 